\title{
Quasi-simultaneous multimodal imaging of cutaneous tissue oxygenation and perfusion
}

Wenqi Ren

Qi Gan

Qiang Wu

Shiwu Zhang

Ronald $\mathrm{Xu}$

\section{SPIE.}




\title{
Quasi-simultaneous multimodal imaging of cutaneous tissue oxygenation and perfusion
}

\author{
Wenqi Ren, ${ }^{a, b}$ Qi Gan, ${ }^{a}$ Qiang Wu, ${ }^{a}$ Shiwu Zhang, ${ }^{a, *}$ and Ronald Xu ${ }^{a, b, *}$ \\ aUniversity of Science and Technology of China, Department of Precision Machinery and Precision Instrumentation, Hefei 230027, China \\ ${ }^{\mathrm{b}}$ The Ohio State University, Department of Biomedical Engineering, Columbus, Ohio 43210, United States
}

\begin{abstract}
Simultaneous and quantitative assessment of multiple tissue parameters may facilitate more effective diagnosis and therapy in many clinical applications, such as wound healing. However, existing wound assessment methods are typically subjective and qualitative, with the need for sequential data acquisition and coregistration between modalities, and lack of reliable standards for performance evaluation or calibration. To overcome these limitations, we developed a multimodal imaging system for quasi-simultaneous assessment of cutaneous tissue oxygenation and perfusion in a quantitative and noninvasive fashion. The system integrated multispectral and laser speckle imaging technologies into one experimental setup. Tissue oxygenation and perfusion were reconstructed by advanced algorithms. The accuracy and reliability of the imaging system were quantitatively validated in calibration experiments and a tissue-simulating phantom test. The experimental results were compared with a commercial oxygenation and perfusion monitor. Dynamic detection of cutaneous tissue oxygenation and perfusion was also demonstrated in vivo by a postocclusion reactive hyperemia procedure in a human subject and a wound healing process in a wounded mouse model. Our in vivo experiments not only validated the performance of the multimodal imaging system for cutaneous tissue oxygenation and perfusion imaging but also demonstrated its technical potential for wound healing assessment in clinical practice. () 2015 Society of Photo-Optical Instrumentation Engineers (SPIE) [DOI: 10.1117/1.JBO.20.12.121307]
\end{abstract}

Keywords: multimodal imaging; multispectral; laser speckle; oxygenation; perfusion; phantom; wound healing.

Paper 150062SSPR received Feb. 2, 2015; accepted for publication Jul. 7, 2015; published online Sep. 10, 2015.

\section{Introduction}

Accurate assessment of cutaneous tissue oxygenation and blood perfusion is important in many clinical applications such as wound healing, ${ }^{1,2}$ diabetes, ${ }^{3,4}$ and plastic surgery. ${ }^{5,6}$ For instance, chronic wounds are among the most common wounds in clinical medicine, greatly reducing patients' quality of life and costing hundreds of billions of dollars for treatment annually. ${ }^{7}$ The normal wound healing process involves the reparative phases of inflammation, proliferation, and remodeling. ${ }^{1}$ During such phases, sufficient oxygen and nutrition delivered by subcutaneous perfusion are critical for successful wound healing. ${ }^{8}$ Because oxygenation and perfusion are significant for understanding the mechanism of chronic wounds and monitoring the wound healing process, accurate and quantitative characterization of these functional parameters will enhance appropriate prevention, assessment, and treatment of chronic wounds.

In the past, many clinical methods and optical systems have been developed to measure tissue functional parameters. Existing methods for tissue oxygenation measurement include the use of polarographic microelectrodes, ${ }^{9}$ transcutaneous oxygen meters, ${ }^{10}$ pulse oximeters, ${ }^{11}$ and hyperspectral imaging. ${ }^{12}$ In addition, current techniques for perfusion measurement include electrohemodynamics, ${ }^{13}$ magnetic resonance imaging, ${ }^{14}$ laser Doppler, ${ }^{15}$ thermodilution, ${ }^{16}$ radiation tracers, ${ }^{17}$ and use of Indocyanine Green dye. ${ }^{18}$ Among existing tools for oxygenation and perfusion measurements, the emerging optical imaging

*Address all correspondence to: Shiwu Zhang, E-mail: swzhang@ustc.edu.cn; Ronald Xu, E-mail: xu.202@osu.edu tools are able to map tissue optical characteristics and functional properties in a rapid, cost effective, and noninvasive fashion. The optical method is always limited in its detection depth. In the ultraviolet-visible range of the spectrum $(<700 \mathrm{~nm})$, light can penetrate tissue by a few hundred microns to a millimeter in depth. Meanwhile, in the near-infrared (NIR) spectral range (700 to $900 \mathrm{~nm}$ ), light can propagate several centimeters in depth due to decreased absorption. ${ }^{19}$ As a new avenue for realtime assessment of tissue oxygenation, multispectral or hyperspectral imaging acquires reflectance images of biologic tissue at multiple wavelengths and reconstructs the oxygen saturation map by a number of algorithms. ${ }^{12,20,21}$ Laser speckle imaging reconstructs a two-dimensional map of blood flow over an area of tissue based on spatial or temporal statistics of the speckle pattern. ${ }^{22}$ Furthermore, several imaging modalities can be integrated in a multimodal imaging system for comprehensive assessment of multiple tissue parameters. ${ }^{23-27}$ However, many tissue parameters acquired by the existing multimodal imaging systems are typically relative and semi-quantitative, and sequential data acquisition is needed for coregistration between individual modalities. ${ }^{28,29}$ Quantitative multimodal imaging is also challenged by the lack of a reliable standard for performance evaluation and calibration, the difficulty of conducting continuous monitoring and rapid recording of tissue dynamic changes, and the measurement disparities resulting from inappropriate coregistration, measurand fluctuations, tissue heterogeneities, and patient-to-patient variations. ${ }^{30}$ 
To overcome these limitations, we developed a multimodal imaging system that shares the same imaging module for quasisimultaneous, quantitative, and noninvasive assessment of cutaneous tissue oxygenation and blood perfusion. The system integrated multispectral and laser speckle imaging technologies into one experimental setup. During a data acquisition session, the individual imaging modalities can be alternated rapidly without moving the imaging module or the target tissue, leading to a significantly simplified postprocessing procedure. Tissue oxygenation and blood perfusion were reconstructed based on a wide gap second derivative reflectometry algorithm and a laser speckle contrast analysis (LASCA) algorithm. The accuracy and reliability of the reconstruction algorithm were quantitatively validated in two calibration experiments in Secs. 2.4.1 and 2.4.2. A skin-simulating phantom was designed to test system performance for measurement of multiple functional parameters. The experimental results were compared with a commercial Moor tissue oxygenation and perfusion monitor. Dynamic detection of cutaneous tissue oxygenation and perfusion was also demonstrated in vivo by a postocclusion reactive hyperemia (PORH) procedure in a healthy human subject and a wound healing process in a wounded mouse model. Our in vivo experiments not only validated the performance of the multimodal imaging system for cutaneous tissue oxygenation and perfusion imaging, but also demonstrated its technical potential for wound healing assessment in clinical practice.

\section{Materials and Methods}

\subsection{Multimodal Imaging System}

The multimodal imaging system integrates multispectral and laser speckle imaging technologies into one setup, as shown in Fig. 1. One advantage of this system is the automatic and rapid switching between two different imaging modalities for simultaneous and dynamic data acquisition in one identical field of view (FOV). As shown in the figure, an acousto-optic tunable filter (AOTF) light source (500 to $900 \mathrm{~nm}$ range, $10 \mathrm{~nm}$ bandwidth at $633 \mathrm{~nm}$, Brimrose) for multispectral imaging and a laser device ( $\lambda=785 \mathrm{~nm}, 150 \mathrm{~mW}$, Changchun New Industries,

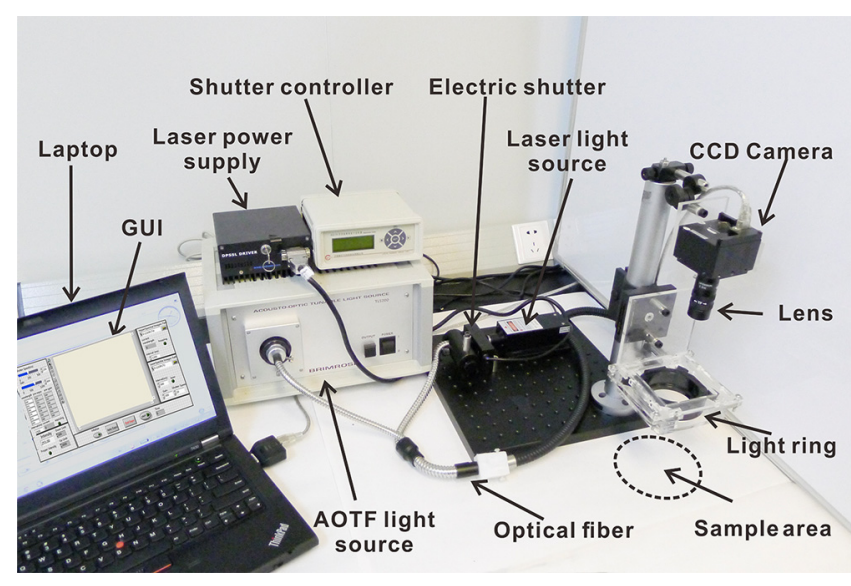

Fig. 1 The simultaneous multimodal imaging system: multispectral light source [acousto-optic tunable filter (AOTF) light source] and laser speckle light source (laser device) are connected to a light ring via a bifurcated optical fiber for average illumination, and an electric shutter is fixed on the fiber to switch two illumination modalities programmatically. The CCD camera will be triggered for data acquisition with corresponding light illumination.
China) for laser speckle imaging are connected to a light ring via a bifurcated optical fiber for uniform illumination. An electric shutter (Daheng New Epoch Technology, Inc., Beijing, China) is fixed on the fiber to alternate between two illumination modalities programmatically. A homochromous 12-bit charge-coupled device (CCD) camera (Microvision Digital Imaging Technology Co. Ltd., China) is used to acquire the reflected intensity of biologic tissue or tissue-simulating phantoms with a resolution of $1392 \times 1040$ pixels. The quantum efficiency of the CCD chip is greater than $40 \%$ at a wavelength range from 400 to $800 \mathrm{~nm}$, suitable for multispectral and laser speckle images collection. The lens can be changed based on the area of samples. The light source and electric shutter are connected to a laptop via serial port for instrument control, and the camera is connected to an image grabber through 1394A port for image transmission. To control the equipment and synchronize data acquisition tasks between modalities, we have developed a graphic user interface (GUI) based on the LabVIEW software platform (National Instruments, Texas).

\subsection{Image Acquisition}

The multimodal imaging procedure was exemplified by a single image acquisition sequence, as described below. In order to obtain a multispectral dataset, a sample to be measured and a 99\% reflected diffuser (National Institute of Standards and Technology, Gaithersburg, Maryland) were placed within the FOV of the CCD camera at an equal height. Then an image was captured in a completely dark environment to record the dark current noise of the CCD chip. Next, the electric shutter was closed to block the $785 \mathrm{~nm}$ laser light path, and the AOTF tunable light source was controlled to illuminate the sample with the specific wavelength beam $(544,552,568,576,592$, and $600 \mathrm{~nm}$ ) successively. At the same time, the autoexposure adjusting function was run to set up the optimal exposure time of each wavelength in the case of improper intensity of the sample. Next, monochromatic images of every wavelength were taken and stored at corresponding exposure times for further processing. To obtain the laser speckle data, the electric shutter was switched to the open position, and the raw speckle images on target sample were acquired at 5 frames/s with the camera exposure time set to be $15 \mathrm{~ms}$. Including sequential exposure, image storage, modality switch, and system delay, a complete sequence of multimodal data collection was performed inapproximately 6 to $8 \mathrm{~s}$. Because the image acquisition sessions at multiple wavelengths were not strictly simultaneous, the time series for each set of multispectral images was interpolated onto a common time base. In addition, to avoid the measurement artifact caused by ambient light, the experiment was carried out in a dark environment.

\subsection{Image Processing}

Image processing includes tissue oxygenation reconstruction from multispectral images with a wide gap second derivative reflectometry algorithm and tissue perfusion reconstruction from laser speckle images with a LASCA algorithm. The processing was performed using MATLAB® software (The Mathworks Incorporated, Natick, Massachusetts).

\subsubsection{Tissue oxygenation reconstruction from multispectral images}

In our system, tissue oxygen saturation $\left(\mathrm{StO}_{2}\right)$ was reconstructed by a second derivative algorithm based on the reflectance 
measurements at six designated wavelengths. ${ }^{31-33}$ Operated at these wavelengths, our imaging system may not be able to reach the same imaging depth as that of an NIR spectroscopic device or an RGB reflectometry device. However, the proposed wavelength set and the imaging algorithm are able to facilitate more sensitive detection of tissue $\mathrm{StO}_{2}$ fluctuations with minimal measurement bias induced by skin condition variations in terms of blood concentration, scattering, and melanin. Furthermore, our imaging system can be configured for multispectral imaging in a broader wavelength range because the AOTF tunable light source has an operation wavelength range of 500 to $900 \mathrm{~nm}$ and the CCD camera is monochromatic with high spectral responsivity and large dynamic range.

The imaging algorithm includes four consecutive steps of calculating absorption-scattering ratio, deriving wide gap second derivative, obtaining analytical expression of second derivative ratio (SDR), and curve fitting for $\mathrm{StO}_{2}$ calculation. In this algorithm, six wavelengths $(544,552,568,576,592$, and $600 \mathrm{~nm}$ ) are screened to calculate an SDR, an index corrected to $\mathrm{StO}_{2}$ regardless of blood concentration, scattering, and melanin level. ${ }^{31}$ SDR can be calculated as

$$
\begin{aligned}
& \operatorname{SDR}(568,576)=\frac{r(592)+r(544)-2 r(568)}{r(600)+r(552)-2 r(576)}, \\
& r(\lambda)=\frac{\mu_{a}(\lambda)}{\mu_{s}^{\prime}(\lambda)}=\frac{s^{2}}{1-s^{2}},
\end{aligned}
$$

where $\mu_{a}$ is the absorption coefficient and $\mu_{s}^{\prime}$ is the reduced scattering coefficient, $s$ and $r(\lambda)$ can be solved using a simplified numerical model reported by Prahl. ${ }^{34}$ The index has a monotonous relationship with $\mathrm{StO}_{2}$ as shown in Eq. (2):

$$
\mathrm{StO}_{2}=100\left(-1.4 \mathrm{SDR}^{3}+4.82 \mathrm{SDR}^{2}-5.66 \mathrm{SDR}+2.38\right) \text {. }
$$

To obtain $\mathrm{StO}_{2}$ from the measurements of SDR, the reflectance map at each wavelength $R(\lambda)$ is calculated by taking the ratio of the skin and the diffuser measurements pixel by pixel. Next, the SDR value is calculated using Eq. (1). Finally, the $\mathrm{StO}_{2}$ map of skin tissue is obtained by Eq. (2).

\subsubsection{Tissue perfusion reconstruction from laser speckle images}

Tissue perfusion characteristics can be acquired with spatial LASCA algorithm. ${ }^{22}$ Illumination of a tissue by monochromatic laser light will produce an interference pattern on the tissue surface. The speckle images exhibit blurring over the exposure time of the camera, which is more pronounced in vascularized regions due to the motion of red blood cells. By analyzing these intensity fluctuations mathematically, parameters about the blood perfusion in the tissue can be obtained.

Next, we present a speckle imaging algorithm modified from that of a Perimed laser speckle imaging system. ${ }^{35}$ In this system, a 785-nm laser device was selected to provide enough depth of light transmission. From the images data, we can obtain the intensity and variance of a region of interest (ROI). To calculate the contrast from intensity-variance pairs, the following equation should be used:

$C=\beta \frac{\sqrt{V}}{I}$, where $C$ is the contrast, $V$ is the variance, $I$ is the intensity, and $\beta$ is the coherence factor. The coherence factor ensures $C=1$ (and thus perfusion $=0$ ) for static objects, which is system specific and will be determined by a calibration experiment as described in Sec. 2.4.2. The perfusion is calculated from the contrast as follows:

$$
P=k\left(\frac{1}{C}-1\right),
$$

where $P$ is the perfusion, $C$ is the contrast, and $k$ is the signal gain factor. Similar to the coherence factor, the signal gain factor will be calibrated to ensure a certain perfusion value on a flux standard.

\subsection{System Verification and Calibration}

Two verification and calibration experiments were conducted, respectively, for multispectral $\mathrm{StO}_{2}$ and laser speckle perfusion measurements.

\subsubsection{Liquid blood phantom experiment for multispectral imaging}

The $\mathrm{StO}_{2}$ algorithm was verified using a liquid blood phantom, ${ }^{30}$ which was prepared by mixing fresh chicken blood, Indian ink (Bomei Biotechnology, Hefei, China), and 20\% intralipid (Sino-Swed Pharmaceutical Corp, Ltd., China) in phosphate buffered solution (Bomei Biotechnology). Ink and intralipid were used to simulate melanin absorption and skin scattering, respectively, and concentration of these ingredients should be determined to ensure that the optical parameters of the phantom would match those of in vivo tissue. In our experiments, the absorption coefficients of the blood phantom were regulated to be $0.172 \mathrm{~cm}^{-1}$ (at $690 \mathrm{~nm}$ ) and the reduced scattering coefficient to be $4.5 \mathrm{~cm}^{-1}$ (at $690 \mathrm{~nm}$ ), as confirmed by an OxiplexTS tissue spectrophotometer (ISS Inc., Champaign, Illinois). The $\mathrm{pH}$ level of the phantom was adjusted to 7.4 by adding $\mathrm{NaOH}$ and $\mathrm{HCl}$. Finally, the oxygenation level of the phantom was adjusted by dropwise addition of sodium hydrosulfite $(0.025 \mathrm{~g} / \mathrm{mL}$, Bomei Biotechnology) using an injection syringe (LSP01-1A, Baoding Longer Precision Pump Co., Ltd., China).

To verify the multispectral imaging system for $\mathrm{StO}_{2}$ measurement, multispectral data of the blood phantom were acquired from the setup shown in Fig. 2. The blood phantom was placed in a container filled with argon gas to eliminate the measurement artifact caused by oxygen in ambient air. During the calibration test, 11 oxygenation levels ranging from nearly $100 \%$ to nearly $0 \%$ were generated by dropwise addition of sodium hydrosulfite at designated doses. At each oxygenation plateau, the blood phantom was mixed to homogeneity by a magnetic stirrer, then multispectral data of each oxygenation level were acquired and saved. At the same time, a probe of the Moor oxygenation monitor (VMS-OXY, Moor Instruments Inc., Devon, UK) was adhered to the beaker, which recorded the actual oxygen saturation of the blood phantom during the calibration procedure.

\subsubsection{Calibration experiment for laser speckle imaging}

The LASCA algorithm was verified by an integrated calibration method using a PFS Flux Standard and a moving white plate. 


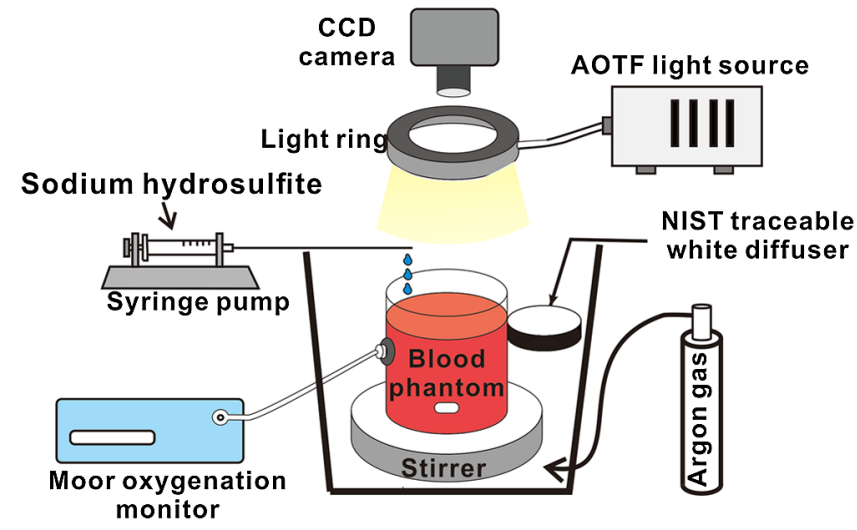

Fig. 2 Schematic drawing of the experimental setup for multispectral imaging of blood phantom. The blood phantom was placed in a container filled with argon gas. A NIST traceable white diffuser was placed next to the phantom for spectrum calibration. Sodium hydrosulfite was added dropwise by a syringe pump for different oxygenation levels. A Moor oxygenation monitor was used to record the actual oxygen saturation of the blood phantom.
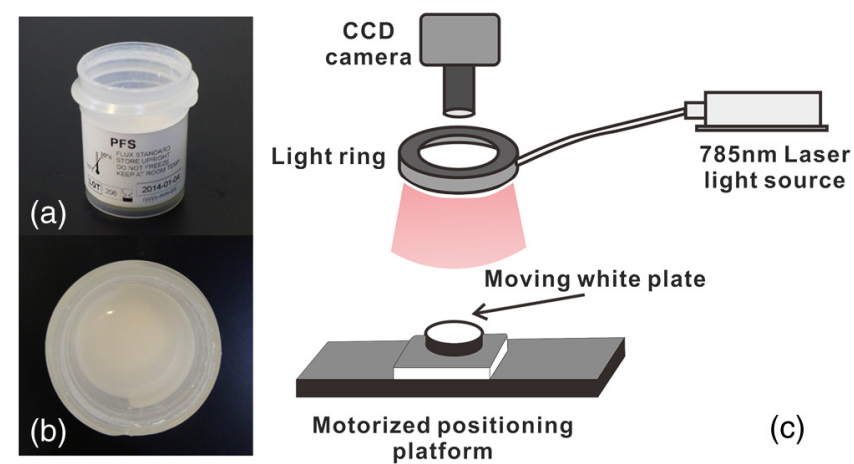

Fig. 3 (a) Picture of a PFS Flux Standard; (b) top view of the PFS Flux Standard; and (c) schematic drawing of the flow simulating experimental setup using a moving white diffuser plate; the plate was pushed by a motorized positioning platform.

The PFS Flux Standard (Moor Instruments Inc., Devon, United Kingdom) is a colloidal suspension of polystyrene particles, as shown in Figs. 3(a) and 3(b). Typically, laser speckle images are acquired after the suspension is shaken gently for $10 \mathrm{~s}$ and rested for $2 \mathrm{~min}$. At room temperature $\left(25^{\circ} \mathrm{C}\right)$, the stationary background area typically produces a contrast value of 1 , and the Flux Standard produces a perfusion value of $250 \pm 5$ Perfusion Units (PUs). Thus, the parameters $\beta$ and $k$ described in Eqs. (3) and (4) can be determined to ensure numeric accuracy.

The linearity of perfusion measurement was verified by a flow-simulating experiment following a previously established protocol. ${ }^{36}$ Figure 3(c) shows the experimental setup for the verification test. A white diffuser plate (National Institute of Standards and Technology) was driven by a motorized positioning system (TSA100, ZOLIX instruments, China) at a constant velocity to simulate the movements of blood flow. The perfusion measurements were taken over a regular velocity range from 0 to $10 \mathrm{~mm} / \mathrm{s}$. At each velocity plateau, 30 consecutive speckle images were acquired at a sampling rate of 5 frames/s. The perfusion $P$ was calculated by LASCA algorithm as described previously.

\subsection{In Vitro Test on a Tissue-Simulating Phantom}

\subsubsection{Tissue-simulating phantom fabrication}

In order to simulate the optical characteristics and the functional parameters of cutaneous tissue, we designed a biocompatible, permanent, and reusable solid phantom that embeds a vesselsimulating channel. The phantom was composed of polydimethylsiloxane (PDMS), titanium dioxide powder $\left(\mathrm{TiO}_{2}\right)$, and Indian ink. First, PDMS (Dow Corning) materials were prepared at a hardener-to-matrix ratio of 1:10 (by weight). Second, $\mathrm{TiO}_{2}$ (Guangfu Fine Chemical Research Institute, China) and Indian ink (Bomei Biotechnology Co., Ltd.) at the designated doses were added into the matrix as the scattering and the absorbing agents, respectively. The mixture was stirred to homogeneity and placed in a vacuum pump for degassing. The mixture was then poured into a rounded mold with a square section vessel model (sectional area $=5 \mathrm{~mm} \times 3 \mathrm{~mm}$ ) placed at the center position. The mixture was kept still for several hours until complete solidification, and the vessel model was then taken out of the phantom. To check the phantom's optical characteristics, an OxiplexTS tissue spectrophotometer was used to confirm the absorption coefficients of PDMS phantom to be $0.122 \mathrm{~cm}^{-1}$ (at $690 \mathrm{~nm}$ ) and the reduced scattering coefficient to be $5.3 \mathrm{~cm}^{-1}$ (at $690 \mathrm{~nm}$ ), and the optical parameters were adjusted to match those of normal human cutaneous tissue. ${ }^{37}$

\subsubsection{Phantom test procedure}

An experimental system as shown in Fig. 4 was established to simulate blood circulation and oxygenation variations. Two completely duplicated PDMS phantoms were connected in series by silicone tubes to avoid optical interference from the Moor monitor during measurement. One phantom was used for multimodal imaging and the other was used to validate the imaging results via an affixed Moor oxygenation and perfusion detector (VMS-OXY and VMS-LDF, Moor Instruments Inc.). A peristaltic pump (BT100-2J, Baoding Longer Precision Pump Co., Ltd.) was used to circulate $250 \mathrm{~mL}$ blood solution (diluted by distilled water in a ratio of 1:5 by volume) for blood

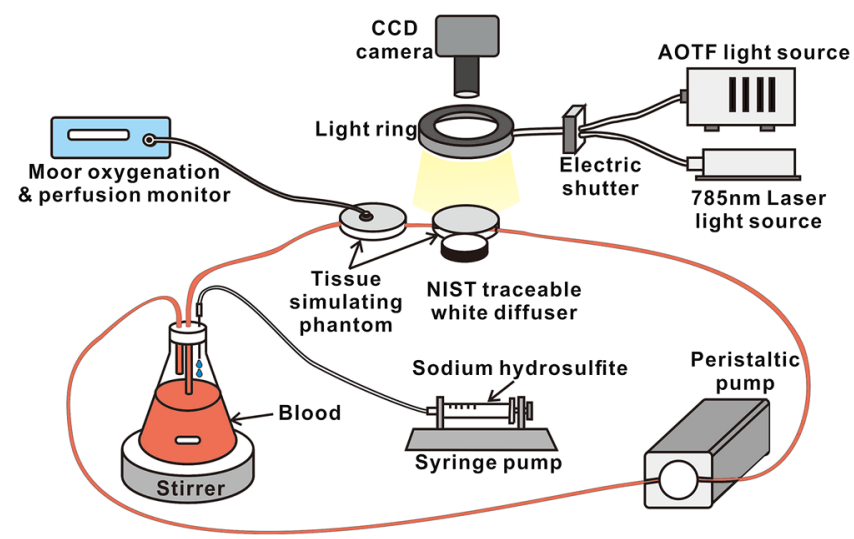

Fig. 4 Schematic drawing of an experimental setup for simulating blood circulation and oxygenation adjustment. Two completely duplicated polydimethylsiloxane (PDMS) phantoms were connected in series for multimodal imaging and Moor monitor comparison. Fresh dilute blood was sealed in a conical flask. In the simulating vessel of the PDMS phantoms, the blood flow was driven by a peristaltic pump and the oxygenation level was adjusted by dropwise addition of sodium hydrosulfite. 
circulation simulation. The blood solution was sealed in a conical flask to eliminate the effect of oxygen in ambient air and weaken the intermittency of the peristaltic pump. The oxygenation level of the blood was adjusted by dropwise addition of sodium hydrosulfite $(0.025 \mathrm{~g} / \mathrm{mL})$ using an injection syringe through a silicone tube. At each oxygenation plateau, the blood phantom was mixed to homogeneity by a magnetic stirrer. Based on this setup, multispectral imaging and laser speckle imaging can be verified on PDMS phantoms simultaneously or consequently.

\subsection{In Vivo Demonstration on Cutaneous Tissue}

\subsubsection{Human skin occlusion test}

The multimodal imaging system of measuring $\mathrm{StO}_{2}$ and perfusion was verified on a healthy volunteer following a similar clinical protocol of PORH as approved by The Ohio State University Institutional Review Board. ${ }^{38}$ The subject's hand was comfortably rested on a countertop within the view field of the CCD camera. A Moor tissue oxygenation and perfusion detector was affixed next to the monitoring region. The PORH process consisted of a preocclusive baseline period (no pressure applied to the arm) of $1 \mathrm{~min}$, a suprasystolic occlusion $(180 \mathrm{~mm}$ $\mathrm{Hg}$ ) period of $3 \mathrm{~min}$, and a reactive hyperemia period (pressure released) of 2 min. During the PORH test, the illumination was switched periodically from multispectral light beam to laser light beam by electric shutter; correspondingly, multispectral images of six designated wavelengths and laser speckle images were acquired. At the same time, Moor oxygenation and perfusion monitor recorded the dynamics of oxygenation and perfusion during the PORH process.

\subsubsection{Nude mouse wound model}

We also established an in vivo mouse dorsal skinfold chamber model to monitor the dynamics of oxygenation and perfusion during the cutaneous wound healing process. ${ }^{39}$ Four male BALB/c-nu nude mice (Vital River Laboratory Animal Technology Co. Ltd., China) of 10 weeks old with a body weight of 23 to $25 \mathrm{~g}$ were used for the experiments. Figure 5(a) shows a nude mouse implanted in a dorsal skinfold chamber; the fold of the depilated dorsal skin of a mouse was taken and then fixed like a sandwich between the two titanium frames of the chamber. A full dermal thickness wound of $2 \mathrm{~mm}$ in diameter was made by removing the skin (including epidermis, dermis, subcutis, cutaneous muscle, and subcutaneous fatty tissue) of one skin layer completely using a biopsy punch. A sterile coverslip was used to close the operation field to avoid infection or mechanical damage. To facilitate multimodal assessment by imaging system, a modified mice holder was developed to fix the mouse as shown in Fig. 5(b). Furthermore, a calibrated white diffuser made of Teflon was used to replace the NIST 99\% reflected diffuser for multispectral imaging as shown in Fig. 5(c). Following acquisition of the multimodal images, a white light reflectance image of the wound area was routinely taken for comparison. The experiments were conducted in accordance with guidelines for the Care and Use of Laboratory Animals and the Institutional Animal Care and Use Committee of University of Science and Technology of China (Protocol No: USTCACUC1401009).

\section{Results and Discussion}

\subsection{System Validation Results}

\subsubsection{Calibration results for multispectral imaging}

At each oxygenation level of each recipe, $\mathrm{StO}_{2}$ was reconstructed from multispectral images using our algorithm as described previously. Three ROIs on the maps were selected to average the $\mathrm{StO}_{2}$ values. After normalization to the scale of $0 \%$ to $100 \%$ by a linear function, the results were compared with the actual $\mathrm{StO}_{2}$ measured by Moor oxygenation monitor. According to Fig. 6(a), linear correlation relationship can be observed between the calculated oxygenation levels and their actual values, indicating that the algorithm is able to reconstruct tissue oxygenation. The measurement error mainly reflects the ambient oxygen interference and different $\mathrm{StO}_{2}$ algorithm between our system and the Moor monitor. Figures 6(b) to 6(e) show the contrast of blood phantom at full oxygenation, deoxygenation, and corresponding $\mathrm{StO}_{2}$ color maps. In these figures the phantom area shows substantial discrimination from deoxygenation to full oxygenation, but the resulting oxygenation value is quite unreasonable in the background area; this reflects the different optical properties of tissue and should be ignored.

\subsubsection{Calibration results for laser speckle imaging}

The calibration procedure for laser speckle blood perfusion imaging consisted of two steps. First, the perfusion result of a Flux Standard and static background was calculated by original LASCA algorithm. Three ROIs marked in Fig. 7(b) were selected to average the contrast and perfusion values. According to Eqs. (3) and (4), to ensure $C=1$ for static background

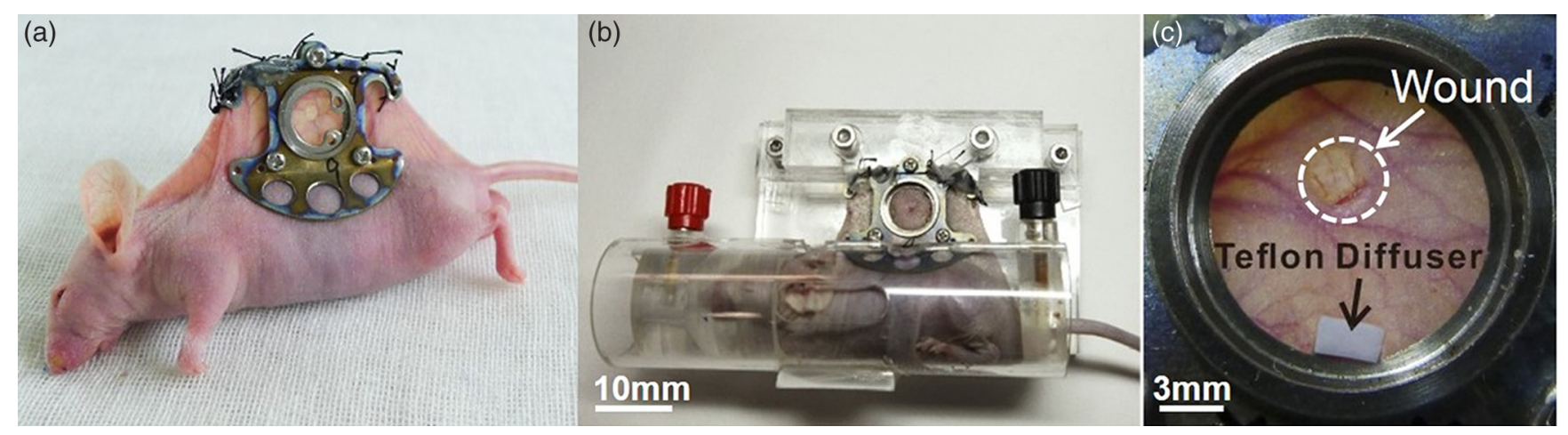

Fig. 5 Nude mice wound model: (a) nude mouse implanted in a dorsal skinfold chamber; (b) a modified mouse holder; and (c) dorsal skinfold wound chamber model with a Teflon diffuser. 

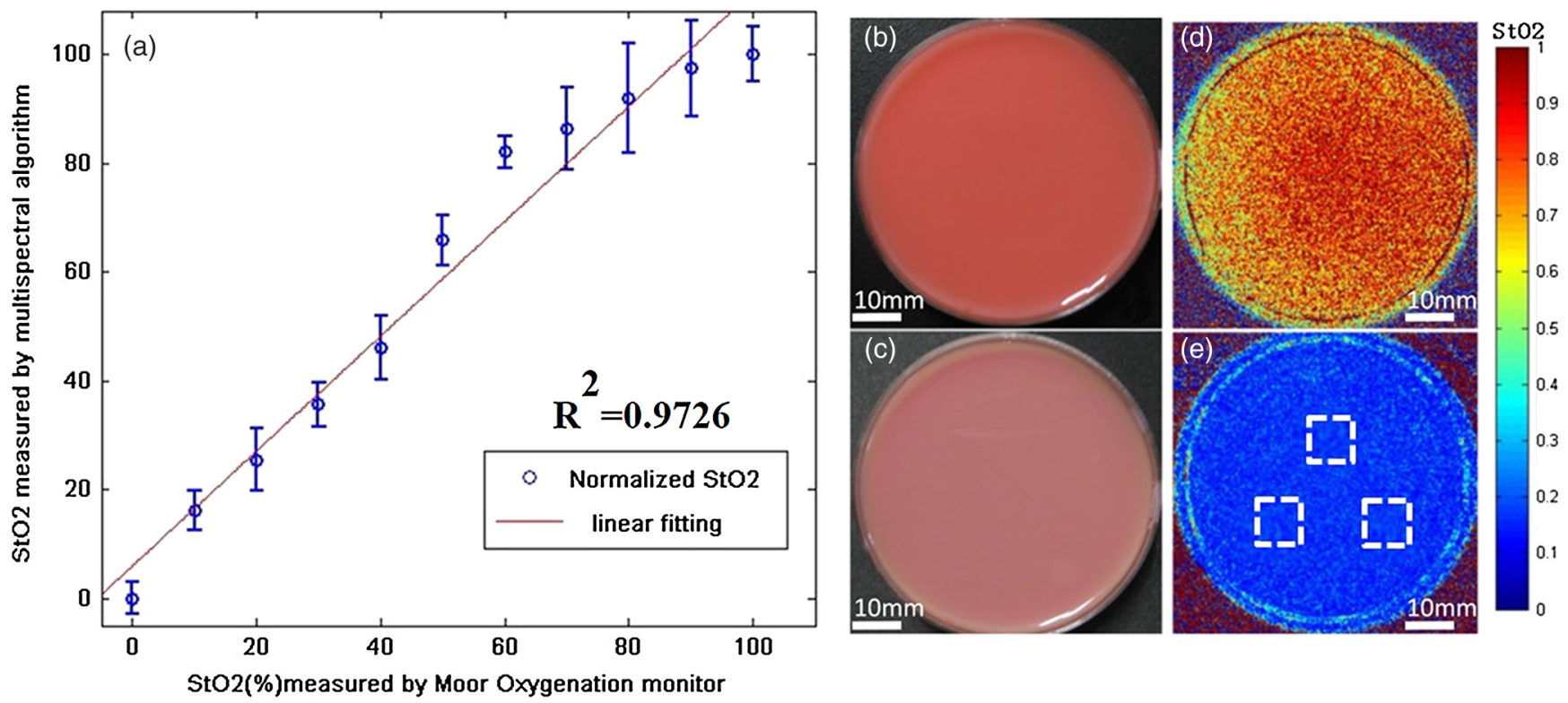

Fig. 6 (a) Blood phantom $\mathrm{StO}_{2}$ measured by multispectral algorithm versus that measured by Moor oxygenation monitor, a good linear correlation relationship can be observed, the linear correlation coefficient $R^{2}=0.9726$. (b) and (c) Blood phantom at full oxygenation and full deoxygenation. (d) and (e) $\mathrm{StO}_{2}$ color maps of blood phantom at full oxygenation and full deoxygenation. The white markers on (e) present three regions of interest (ROIs) selected to average the $\mathrm{StO}_{2}$ values.

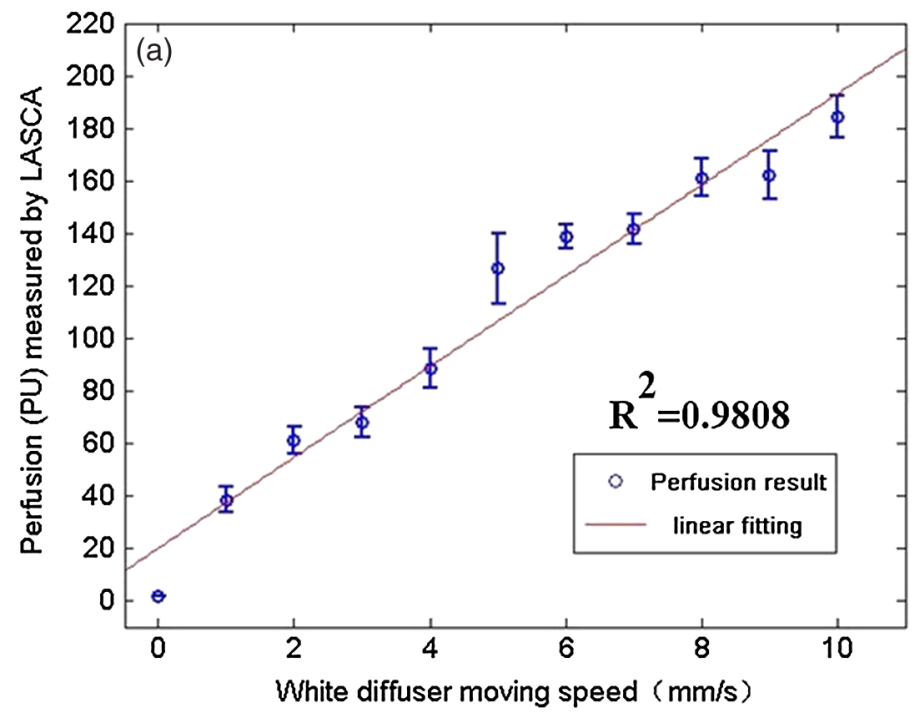

(b)

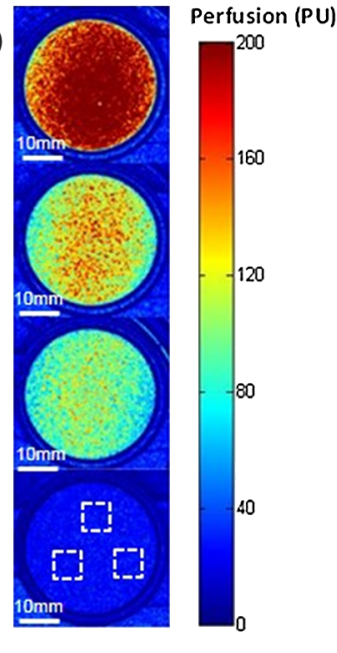

Fig. 7 (a) Perfusion result measured by laser speckle contrast analysis (LASCA) versus white plate moving speed, the linear correlation coefficient is $R^{2}=0.9808$. (b) Perfusion color maps of the moving plate at $0,3,7$, and $10 \mathrm{~mm} / \mathrm{s}$ from bottom to top, respectively. The white markers on the bottom of (b) present the three ROls selected to average the contrast and perfusion values.

and $P=250 \pm 5$ PU for Flux Standard, the parameters $\beta$ and $k$ were determined to be 2.00 and 21.45 , respectively. Second, as shown in Fig. 7(a), perfusion produced from the dynamic speckle on the white diffuser increases linearly with the moving velocity within a regular range of blood flow. The fitting line reveals an impressive linear correlation between two parameters. Figure 7(b) shows four representative perfusion maps at different moving speeds. By combining the results of these two experiments, the linearity and numerical accuracy of laser speckle imaging can be verified.

\subsection{In Vitro Test Results on a Tissue-Simulating Phantom}

A PDMS phantom test using the experimental setup in Fig. 4 was conducted to verify the imaging system in two modalities together. Figures 8(a) and 8(b) demonstrate the RGB pictures of two series-connected, tissue-simulating phantoms and the attached probe of Moor oxygenation and perfusion monitor. As shown in Fig. 8(c), when the peristaltic pump is on, the blood will be driven and the perfusion map shows a high level in the 

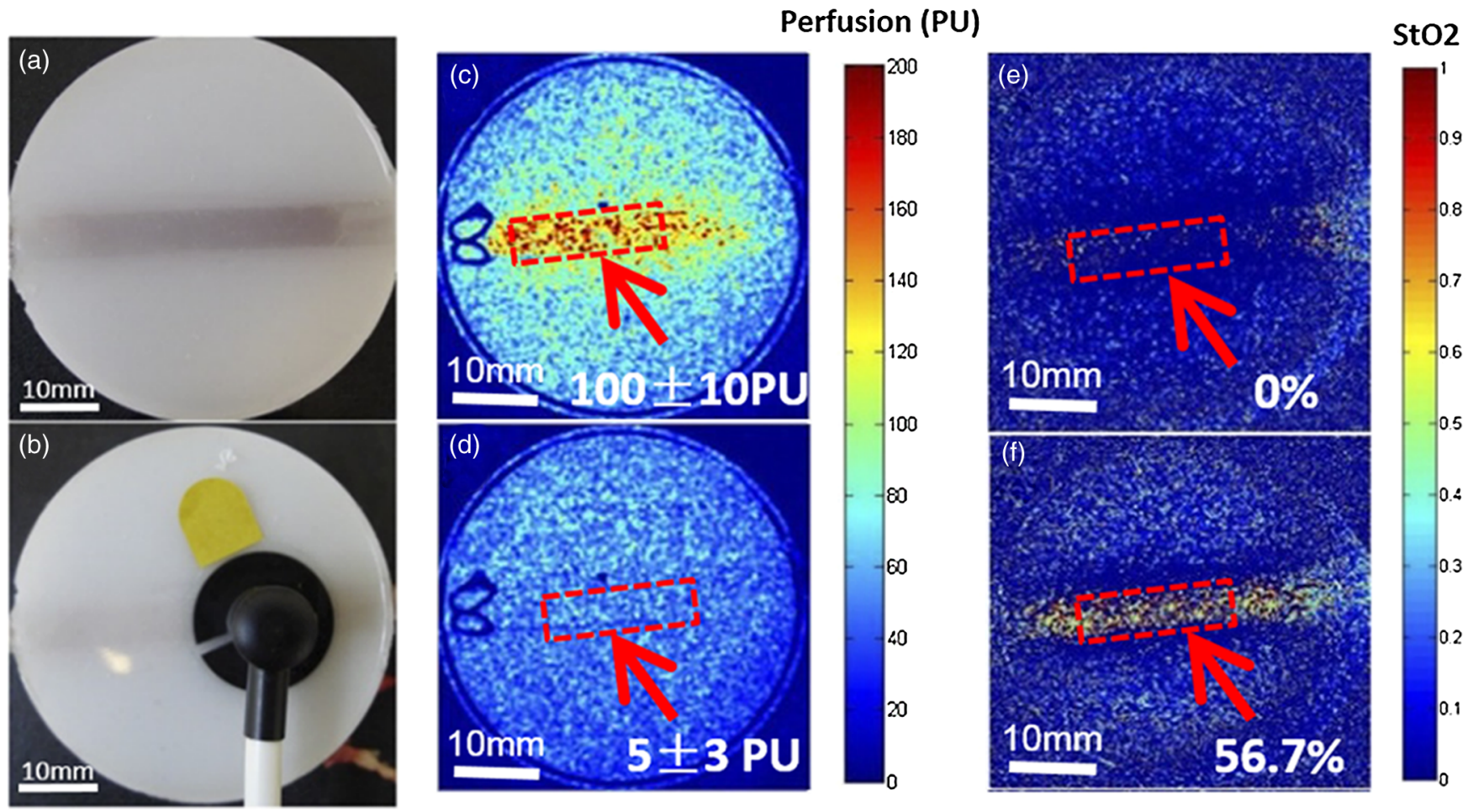

Fig. 8 Pictures and imaging results of PDMS tissue-simulating phantom: (a) The phantom used for multimodal imaging; (b) the phantom used for verification using Moor oxygenation and perfusion monitor; (c) and (d) perfusion maps of the phantom at high blood flow and stationary blood flow; and (e) and (f) oxygenation maps of the phantom at full deoxygenation and full oxygenation. The red markers on (c)-(f) indicate the corresponding detection area of Moor probe on the phantom.
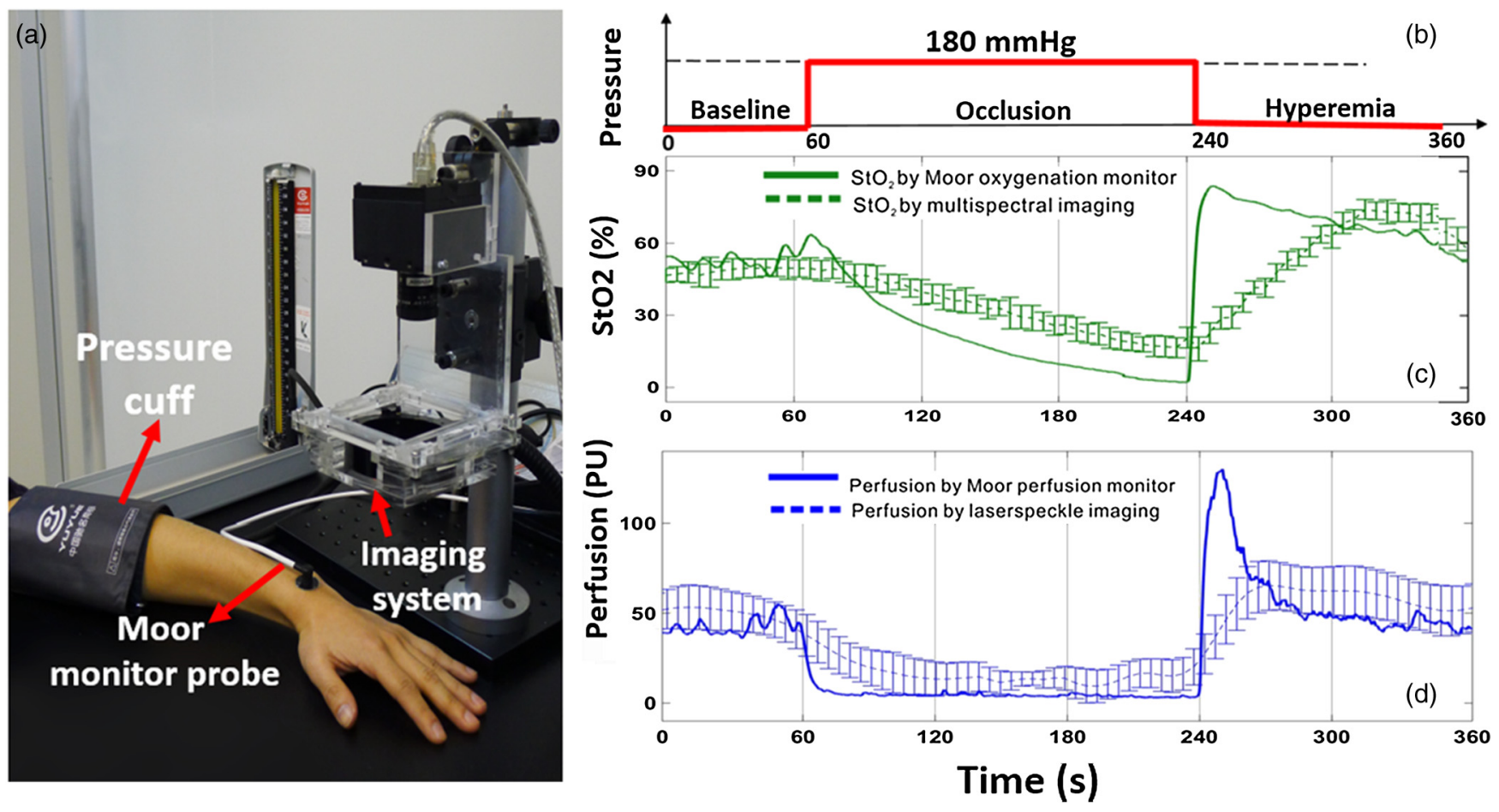

Fig. 9 (a) The subject's hand is comfortably rested on a countertop within the field of view (FOV) of the camera. A Moor tissue oxygenation and perfusion probe is affixed next to the monitoring region. (b) The test protocol consists of a baseline period of $1 \mathrm{~min}$, an occlusion period of $3 \mathrm{~min}$, and then a reactive hyperemia period of 2 min. (c) $\mathrm{StO}_{2}$ measurement results by imaging system and Moor monitor. (d) Perfusion measurement results by imaging system and Moor monitor. 
phantom vessel area; the Moor perfusion monitor displays $100 \pm 10$ PU. As a comparison, Fig. 8(d) shows the perfusion map when blood flow is static, where the Moor monitor displays $5 \pm 3$ PU. The blurry perfusion mapping is mainly due to the scattering medium existing in blood and phantom. Regarding the oxygen saturation adjustment, $\mathrm{StO}_{2}$ maps of the phantom at full deoxygenation and full oxygenation are shown in Figs. 8(d) and 8(e). Accordingly, the Moor oxygenation monitor displays the oxygen saturation as $0 \%$ and $56.7 \%$. One can see that the spatial resolution of $\mathrm{StO}_{2}$ maps is not quite detailed, the most significant reason being the limited transmission depth of the multispectral light source through the simulating phantom. An advanced denoising algorithm may be helpful to optimize results. However, preliminary test results reveal the potential of this phantom to verify our multimodal imaging system.

\subsection{In Vivo Test on Cutaneous Tissue}

\subsubsection{Human skin occlusion test results}

The multimodal imaging system was verified in vivo by human subject testing. Figures 9(a) and 9(b) illustrate the test scene and the protocol of PORH procedure. Figure 9(c) plots the corresponding dynamic changes of skin tissue $\mathrm{StO}_{2}$ and perfusion calculated by averaging three selected ROIs on the monitoring region. It also plots the measurement results of

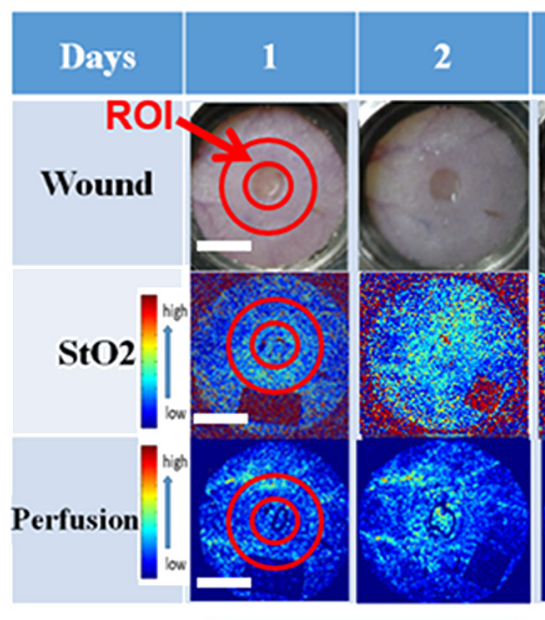

(a)
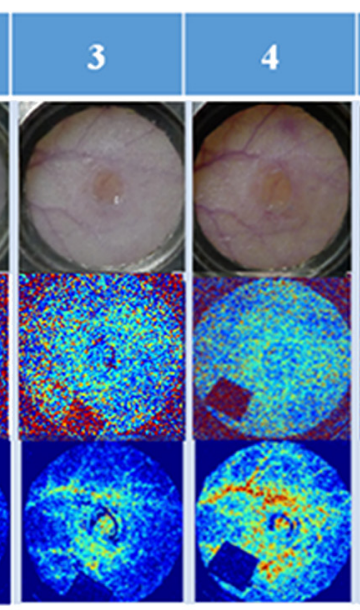

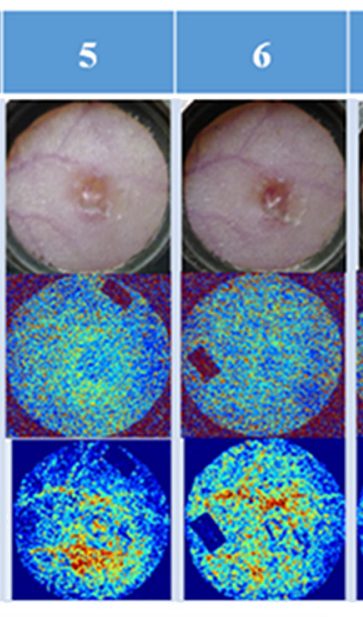

Angiogenesis
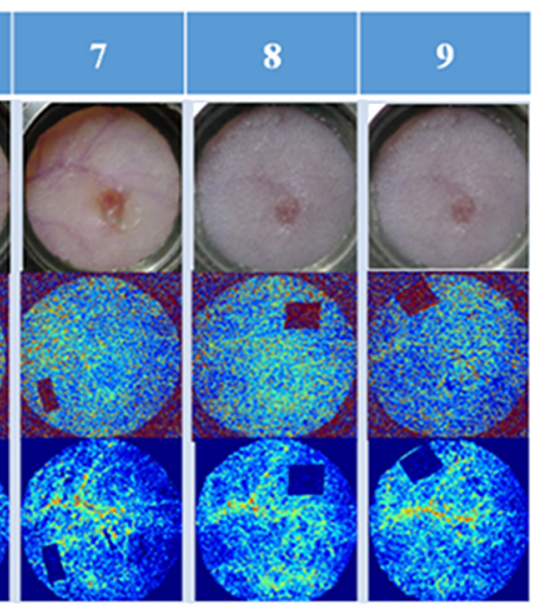

Coalescence
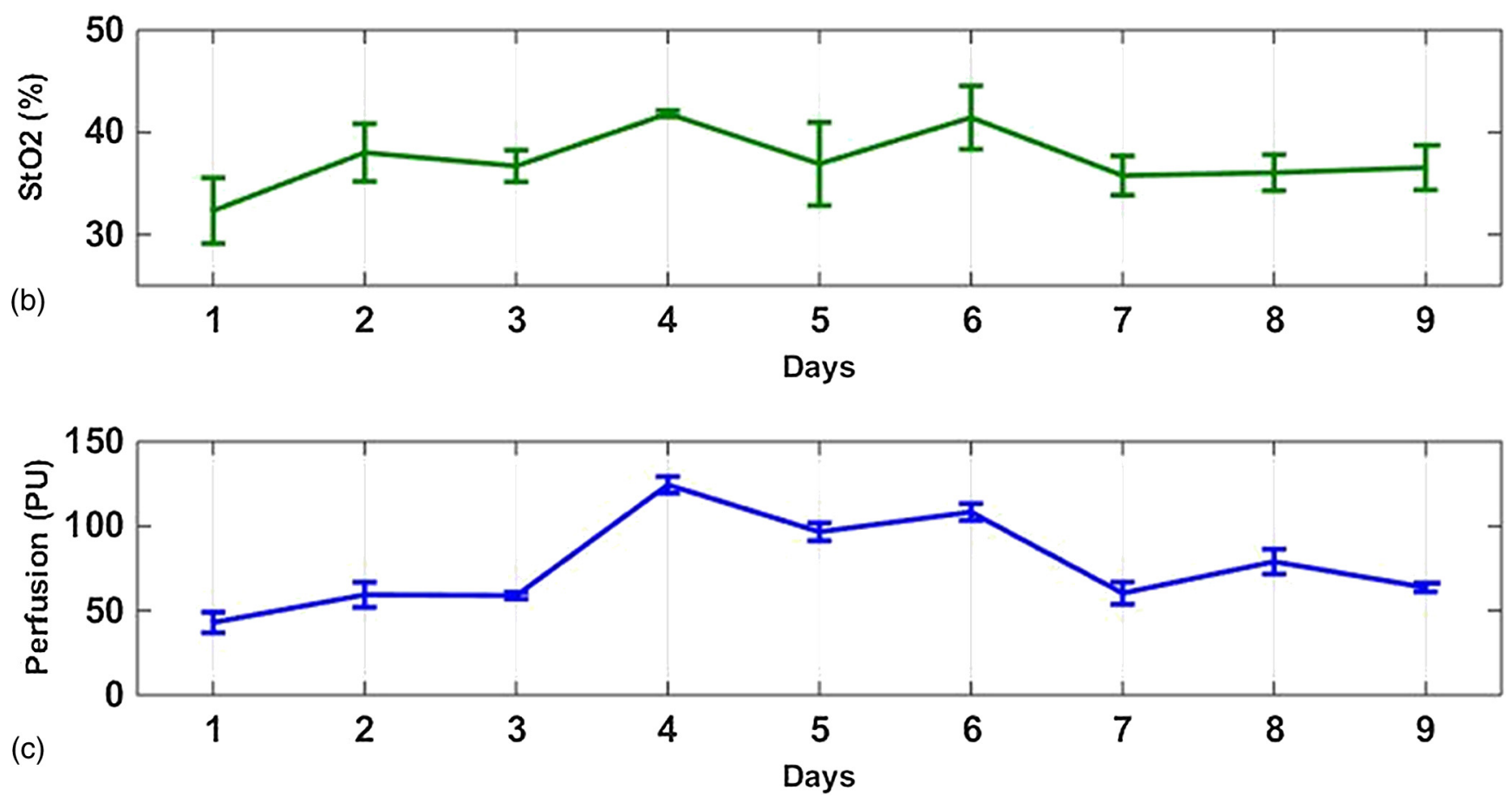

Fig. 10 Results of wound healing monitoring: (a) dynamic maps of wound real-color images (first row), $\mathrm{StO}_{2}$ (second row), and perfusion (last row) of tissue in wound healing process from the first day after biopsy to the ninth day. In the first column, the red concentric circles indicate the annular computational ROls of $\mathrm{StO}_{2}$ and perfusion in the wound periphery. The length of the scale bar is $5 \mathrm{~mm}$. (b) and (c) The dynamics of $\mathrm{StO}_{2}$ and perfusion intensity of ROls in the annular area during wound healing process, respectively. 
Moor oxygenation and perfusion monitor during the PORH process. It can be seen from the green curves that the $\mathrm{StO}_{2}$ measurement results of both the imaging system and the Moor monitor are well-matched. The $\mathrm{StO}_{2}$ level drops during the suprasystolic occlusion period (from the 60th second to the 240th second) and rises above the starting point when the pressure on the arm is released (from the 240th second to the 360th second). The perfusion measurements show similar trends, as plotted by the blue curves in Fig. 9. However, some disparities exist between the two measurements at the initial and the final stages. The disparities may be caused by two reasons. First, the acquisition period of multimodal images took about 6 to $8 \mathrm{~s}$ due to the responsive time of the electric shutter switching, allowing enough exposure time for capturing every image, and the time delay of Labview Run-Time software package. Thus, optimization of the software and reduction of the time required for illumination switch will improve accuracy of measurements. Second, the measurement position and depth of Moor monitor did not coincide exactly with the imaging system. In addition, the Moor monitor may be affected by the variation of blood concentration, skin color, and scattering properties of the human subject.

\subsubsection{Nude mice wound healing process monitoring}

The imaging system was also verified in a nude mice wound model to demonstrate its technical potential for wound healing assessment in clinical practice. Oxygen saturation and blood perfusion maps of the wound periphery were reconstructed and compared. In Fig. 10(a), longitudinal monitoring results of spatiotemporal hemodynamic changes in a mouse wound model are enumerated. The first row shows real-color pictures of the wound area from the first day after biopsy to the ninth day, when the wound had remodeled completely. The second and third rows show the dynamics of $\mathrm{StO}_{2}$ and perfusion maps, respectively. In order to quantify the functional parameter changes during the wound healing progress, annular computational ROIs around the wound were selected. Both $\mathrm{StO}_{2}$ and perfusion intensities have been calculated and plotted as shown in Figs. 10(b) and 10(c). The measurements were triplicated to minimize random errors. Considering the intuitional $\mathrm{StO}_{2}$ maps and the dynamics plot, tissue oxygen saturation at the first day is indicated to be a relatively low level. However, an obvious increase is witnessed from day 2 to day 6 after biopsy. After that, the $\mathrm{StO}_{2}$ subsides slowly and reaches a stable oxygen level as healing is completed. Toward the perfusion, a similar dynamic trend can be seen. The initial perfusion intensity is relatively low, and a significant increase is shown from day 2 to day 6 . On days 7 to day 9 the perfusion is observed to be lower, suggesting a return toward baseline.

Classical physiology of wound healing has been reported by Strodtbeck, ${ }^{40}$ Singer and Clark, ${ }^{8}$ and Ueno et al. ${ }^{41}$ Three phases of hemostasis, angiogenesis, and coalescence can be preliminarily witnessed in these maps. Physiologically speaking, the initial dip in $\mathrm{StO}_{2}$ and perfusion may be attributed to the hemostasis due to biopsy. Schreml et al. ${ }^{42}$ referred that the vascular disruption and vasoconstriction caused a hypoxic microenvironment that was intensified by increased oxygen consumption due to metabolically active cells contributing to wound healing, which also explained the drop in the first days. Also, with the growth of vessels at 3 to 6 days, the parameters exhibited a significant increase, and the results were consistent with our current understanding of the positive and negative regulations of angiogenesis during the different phases of wound healing. ${ }^{43,44}$ Another important mechanism in this stage was activation of vasoactive substances, which increased perfusion to the wound site, whereas the oxygen delivery increased. ${ }^{40}$ Although the mapping results are preliminary and rough in spatial resolution, the system still reveals its capability for simultaneous, dynamic multimodal imaging of tissue oxygenation and perfusion.

\section{Conclusions}

In this paper, we present a multimodal imaging system that integrates multispectral imaging and laser speckle imaging technologies together. The multimodal imaging system utilized only a single camera to realize quasisimultaneous and quantitative characterization of tissue oxygenation and perfusion. Rapid and automatic switching between different modalities makes it possible to obtain dynamic measurement with no need of image coregistration. To validate the imaging system, two quantitative calibration experiments and a new style of skin-simulating phantom were designed; therefore, the numerical accuracy and reliability of the imaging system can be assured. In vivo, a PORH procedure test on a human hand demonstrated the capability of the system for dynamic detection of cutaneous tissue oxygenation and perfusion, and commendable results were witnessed with the comparison to a Moor oxygenation and perfusion monitor. Furthermore, an ongoing wound healing monitoring experiment using nude mice dorsal skinfold chamber models preliminarily revealed the dynamic tendency of $\mathrm{StO}_{2}$ and perfusion parameters during the healing process. All these results not only validated the performance of the multimodal imaging system for cutaneous tissue oxygenation and perfusion imaging, but also demonstrated its technical potential for wound healing assessment in clinical practice. The future work will focus on enhancing the accuracy and processing speed of the system and developing a portable device for multimodal imaging.

\section{Acknowledgments}

The project was partially supported by Natural Science Foundation of China (Nos. 81271527 and 81327803) and the Fundamental Research Funds for the Central Universities (WK2090090013). The authors are grateful to Dr. David Allen of the National Institute of Standards and Technology, who generously provided us the diffusion standard and helpful suggestions.

\section{References}

1. C. K. Sen, "Wound healing essentials: let there be oxygen," Wound Repair Regen. 17(1), 1-18 (2009).

2. G. Broughton, II, J. E. Janis, and C. E. Attinger, "Wound healing: an overview," Plast. Reconstr. Surg. 117(7 Suppl), 1e-S-32e-S (2006).

3. R. L. Greenman et al., "Early changes in the skin microcirculation and muscle metabolism of the diabetic foot," Lancet 366(9498), 1711-1717 (2005).

4. P. E. James et al., "Vasorelaxation by red blood cells and impairment in diabetes reduced nitric oxide and oxygen delivery by glycated hemoglobin," Circ. Res. 94(7), 976-983 (2004).

5. H. W. Hopf et al., "Wound tissue oxygen tension predicts the risk of wound infection in surgical patients," Arch. Surg. 132(9), 997-004 (1997).

6. E. P. Kindwall, L. J. Gottlieb, and D. L. Larson, "Hyperbaric oxygen therapy in plastic surgery: a review article," Plast. Reconstr. Surg. 88(5), 898-908 (1991). 
7. C. K. Sen et al., "Human skin wounds: a major and snowballing threat to public health and the economy," Wound Repair Regen. 17(6), 763-771 (2009).

8. A. J. Singer and R. A. Clark, "Cutaneous wound healing," N. Engl. J. Med. 341(10), 738-746 (1999).

9. N. Chang et al., "Direct measurement of wound and tissue oxygen tension in postoperative patients," Ann. Surg. 197(4), 470 (1983).

10. A. Scheffler and H. Rieger, "Clinical information content of transcutaneous oxymetry $\left(\mathrm{tcpO}_{2}\right)$ in peripheral arterial occlusive disease (a review of the methodological and clinical literature with a special reference to critical limb ischaemia)," Vasa 21(2), 111-126 (1991).

11. S. J. Barker and K. K. Tremper, "Pulse oximetry: applications and limitations," Int. Anesthesiol. Clin. 25(3), 155-175 (1987).

12. S. Shah et al., "Cutaneous wound analysis using hyperspectral imaging," Biotechniques 34(2), 408-413 (2003).

13. L. Djordjevich and M. S. Sadove, "Basic principles of electrohaemodynamics," J. Biomed. Eng. 3(1), 25-33 (1981).

14. R. Luypaert et al., "Diffusion and perfusion MRI: basic physics," Eur. J. Radiol. 38(1), 19-27 (2001).

15. F. Khan and D. J. Newton, "Laser Doppler imaging in the investigation of lower limb wounds," Int. J. Lower Extremity Wounds 2(2), 74-86 (2003).

16. V. Ganz et al., "Measurement of blood flow in the femoral artery in man at rest and during exercise by local thermodilution," Circulation 30(1), 86-89 (1964).

17. L. L. Johnson and D. W. Seldin, "Clinical experience with technetium99m teboroxime, a neutral, lipophilic myocardial perfusion imaging agent," Am. J. Cardiol. 66(13), E63-E67 (1990).

18. V. V. Kupriyanov, S. Nighswander-Rempel, and B. Xiang, "Mapping regional oxygenation and flow in pig hearts in vivo using near-infrared spectroscopic imaging," J. Mol. Cell. Cardiol. 37(5), 947-957 (2004).

19. C. Balas, "Review of biomedical optical imaging - a powerful, noninvasive, non-ionizing technology for improving in vivo diagnosis," Meas. Sci. Technol. 20(10), 104020 (2009).

20. I. Seo, P. R. Bargo, and N. Kollias, "Simultaneous assessment of pulsating and total blood in inflammatory skin lesions using functional diffuse reflectance spectroscopy in the visible range," J. Biomed. Opt. 15(6), 060507 (2010).

21. A. Vogel et al., "Using noninvasive multispectral imaging to quantitatively assess tissue vasculature," J. Biomed. Opt. 12(5), 051604 (2007).

22. J. D. Briers and S. Webster, "Laser speckle contrast analysis (LASCA): a nonscanning, full-field technique for monitoring capillary blood flow," J. Biomed. Opt. 1(2), 174-179 (1996).

23. J. H. Klaessens et al., "Multimodal tissue perfusion imaging using multi-spectral and thermographic imaging systems applied on clinical data," Proc. SPIE 8574, 85740F (2013).

24. P. B. Jones et al., "Simultaneous multispectral reflectance imaging and laser speckle flowmetry of cerebral blood flow and oxygen metabolism in focal cerebral ischemia," J. Biomed. Opt. 13(4), 044007 (2008).

25. A. K. Dunn et al., "Simultaneous imaging of total cerebral hemoglobin concentration, oxygenation, and blood flow during functional activation," Opt. Lett. 28(1), 28-30 (2003).

26. A. Steimers et al., "Simultaneous imaging of cortical haemoglobin concentration and blood flow with RGB reflectometry and LASCA during cortical activation in rats," Proc. SPIE 8804, 880403 (2013).

27. N. Yokoi et al., "Imaging of blood flow and blood concentration change in a frame rate using laser speckle: methods for image analysis," Opt. Laser Technol. 64, 352-362 (2014).

28. S. Zhang et al., "Multimodal imaging of ischemic wounds," Proc. SPIE 8553, 85531G (2012).

29. R. X. Xu et al., "Dual-mode imaging of cutaneous tissue oxygenation and vascular function," J. Vis. Exp. (46) (2010).

30. R. X. Xu et al., "Developing digital tissue phantoms for hyperspectral imaging of ischemic wounds," Biomed. Opt. Express 3(6), 1433-1445 (2012).

31. J. Huang, "Multispectral imaging of skin oxygenation," $\mathrm{PhD}$ Thesis, The Ohio State University (2012).
32. D. E. Myers et al., "Noninvasive method for measuring local hemoglobin oxygen saturation in tissue using wide gap second derivative nearinfrared spectroscopy," J. Biomed. Opt. 10(3), 034017 (2005).

33. J. Huang et al., "A second derivative multispectral algorithm for quantitative assessment of cutaneous tissue oxygenation," J. Biomed. Opt. 20(3), 036001 (2015).

34. S. A. Prahl, "Simple and accurate approximations for reflectance from a semi-infinite turbid medium," in OSA Biomedical Topical Meetings, pp. 613-614 (2002).

35. Perimed, "Real-time microcirculation imaging," http://www.perimedinstruments.com/upl/files/103723/PeriCam_PSI_System_laser_speckle_ blood_perfusion_imager_Perimed.pdf (1 August 2015)

36. P. Li et al., "Imaging cerebral blood flow through the intact rat skull with temporal laser speckle imaging," Opt. Lett. 31(12), 1824-1826 (2006).

37. T. Lister, P. A. Wright, and P. H. Chappell, "Optical properties of human skin,” J. Biomed. Opt. 17(9), 090901 (2012).

38. H. Arkin, L. Xu, and K. Holmes, "Recent developments in modeling heat transfer in blood perfused tissues," IEEE Trans. Biomed. Eng. 41(2), 97 (1994).

39. H. Sorg, C. Krueger, and B. Vollmar, "Intravital insights in skin wound healing using the mouse dorsal skin fold chamber," J. Anat. 211(6), 810-818 (2007).

40. F. Strodtbeck, "Physiology of wound healing," Newborn Infant Nurs. Rev. 1(1), 43-52 (2001).

41. C. Ueno, T. K. Hunt, and H. W. Hopf, "Using physiology to improve surgical wound outcomes," Plast. Reconstr. Surg. 117(7 Suppl), 59S$71 \mathrm{~S}$ (2006).

42. S. Schreml et al., "Oxygen in acute and chronic wound healing," Br. J. Dermatol. 163(2), 257-268 (2010).

43. A. Rege et al., "In vivo laser speckle imaging reveals microvascular remodeling and hemodynamic changes during wound healing angiogenesis," Angiogenesis 15(1), 87-98 (2012).

44. M. Schafer and S. Werner, "Cancer as an overhealing wound: an old hypothesis revisited," Nat. Rev. Mol. Cell Biol. 9(8), 628-638 (2008).

Wenqi Ren is a PhD student at the University of Science and Technology of China and a visiting scholar at The Ohio State University. His research interests include biomedicaloptics, multimodal imaging, and portable medical imaging devices.

Qi Gan is a MS student at the University of Science and Technology of China. His research interests include biomedical optics and surgical navigation.

Qiang $\mathbf{W u}$ is a PhD student at the University of Science and Technology of China. His research interests include microencapsulation for drug delivery and medical device design. He is a student member of SPIE.

Shiwu Zhang is currently an associate professor in the Department of Precision Machinery and Precision Instrumentation, USTC. He has also been a postdoctoral research fellow in the Department of Computer Science, Hong Kong Baptist University, Hong Kong. He has led a number of research programs sponsored by the National Science Foundation of China, Chinese Academy of Sciences, Chinese State High-Tech Development Plan, and others. His current research interests include biomedical optics and intelligent robotics.

Ronald $\mathbf{X u}$ is an associate professor of biomedical engineering at The Ohio State University and a professor of precision machinery and instrumentation at the University of Science and Technology of China. He has published over 80 technical papers in biomedical imaging, drug delivery, and 3-D printing. In 2010, he was honored as 1 of the 10 brightest Central Ohioans by Columbus CEO Magazine. In 2011, he was awarded the TechColumbus "Inventor of the Year" Award. 